\title{
New Fiber Matrix Process with 3D Fiber Printer - A Strategic In-process Integration of Endless Fibers Using Fused Deposition Modeling (FDM)
}

\author{
Andreas Fischer, Steve Rommel, and Thomas Bauernhansl \\ Fraunhofer Institute for Manufacturing Engineering and Automation, \\ Nobelstrasse 12. 70569 Stuttgart, Germany \\ \{andreas.fischer, steve.rommel\}@ipa.fraunhofer.de
}

\begin{abstract}
Product manufacturers are faced with a constant decrease in product development time, short product life cycles and an increase of complexity of the products. At the same time, it is becoming more and more difficult to justify the expected product features while satisfying the requirements of the trends like eco-design, resource efficiency, light weight design and individualization. These developments are key characteristics which favor the growing role and importance of additive manufacturing (AM). Additive Manufacturing techniques such as Fused Deposition Modeling (FDM), Selective Laser Sintering (SLS) or Selective Laser Melting (SLM) are transitions from building prototypes to manufacturing series products within short time and with highest requirements regarding material characteristics as well as product performance. Product performance is one of the main drivers of the creation of multi-material composites. These composites, existing of a matrix and embedded fibers, are more and more used in various industries not just the aerospace and automotive industry. They offer a solution to the light-weight requirements amongst others. Yet, so far the manufacturing of composites is only partially automated; it requires a lot of manual labor and is typically requiring tools resulting in added costs. A combination or integration of additive and composite manufacturing is very limited to not existing, being an indicator for the difficulties to bring these two technologies together.

To meet this challenge the 3D Fiber Print has been developed. It is an AM method using Fused Deposition Modeling (FDM) and composites in process simultaneously while manufacturing a product. The products are still manufactured layer-wise, with each layer containing a composite on-demand. The newly developed and patented print head is designed in the way that composite and matrix material are applied in the right setup and ration using a nozzle style print head to completely embed the composite. This paper presents the setup and possibilities of this new application.
\end{abstract}

Keywords: Additive Manufacturing, Fused Deposition Modeling, Fibers. 


\section{Introduction to Additive Manufacturing}

Additive manufacturing is a technology that is considered to have the potential to start a new industrial revolution. Mostly known as rapid prototyping a variation of terms is used for "additive manufacturing" depending on the purpose of the product:

- Rapid Prototyping (RP) - building sample parts and prototypes

- Rapid Tooling (RT) - building tools for other manufacturing methods

- "Generative Manufacturing" or Additive Manufacturing (AM) - end-products with small- to medium-sized quantities.

The various technologies within additive manufacturing differentiate from conventional manufacturing technologies in the strategy the parts are produced. Contrary to CNC milling for example, being a subtracting manufacturing method and therefore manufacturing a product by removing material from a block, an additive manufactured product is built by placing material layer by layer onto each other until the final part shape is completed. Each layer is solidified individually, but also adhered to the ones next to it to form a continuous shape. Adhering these layers to each other is achieved by introducing an energy force, typically in the form of heat or light. This principle of building a product and its advantages is also the biggest disadvantage. The ,created border” between these layers is a potential weak point within the product itself when it comes to mechanical or thermal stresses during use. One way to overcome this issue is taking precautions in the design and construction of a product following the stress analysis and strengthening the part where needed. This approach by itself is common to conventional design methods. Additionally the placement of the product to be built in the build space of the machine and therefore its orientation is also one way to reduce the risk of failure.

It can be argued that the advantages as well as the disadvantages of additive manufacturing result from the reduction of a three-dimensional manufacturing issue to a twodimensional layerwise manufacturing issue. The selection of a manufacturing strategy or method and its corresponding planning process of conventional manufacturing are no longer applicable when it comes to additive manufacturing. The planning process can be considered lean. Benefits of this circumstance are saving in time and cost in the case of an increase in product complexity. Considering time and cost it is also true that an increase in detail by reducing the layer thickness or an increase in product size are typically resulting in a significant increase in production or building time.

Besides the classification of the additive manufacturing following DIN 8580 the following criteria can be used to classify additive manufacturing:

- Physical state of material

- Material the product is made of

- Usage of products within the product development process

- Manufacturing principle

One of the best describing classifica-tions is probably the manufacturing principle as shown in the table below. 
Table 1. Classification of Additive Manufacturing according to the manufacturing principle

\begin{tabular}{|c|c|c|c|}
\hline Principle group & AM & Abreviation & principle \\
\hline \multirow[t]{3}{*}{ Sintering } & Selective Laser Sintering & SLS & \multirow{3}{*}{$\begin{array}{c}\text { localized } \\
\text { powder melting }\end{array}$} \\
\hline & Selective Laser Melting & SLM & \\
\hline & Electron Beam Melting & EBM & \\
\hline \multirow[t]{2}{*}{ Extrusion } & $\begin{array}{l}\text { Fused Deposition } \\
\text { Modeling }\end{array}$ & FDM & \multirow{2}{*}{$\begin{array}{c}\text { Application of } \\
\text { liquified polymers } \\
\text { using nozzles }\end{array}$} \\
\hline & Multi-Jet-Modeling & MJM & \\
\hline UV-Hardening & Stereolithographie & SLA & $\begin{array}{c}\text { Localized } \\
\text { co-polymerisation }\end{array}$ \\
\hline $\begin{array}{c}\text { Binder } \\
\text { Technology }\end{array}$ & 3-Dimensional Printing & 3DP & $\begin{array}{c}\text { Localized Application } \\
\text { of binder }\end{array}$ \\
\hline Laminating & $\begin{array}{l}\text { Laminated Object Manufac- } \\
\text { turing }\end{array}$ & LOM & $\begin{array}{l}\text { Contouring and apply- } \\
\text { ing of sheet material }\end{array}$ \\
\hline
\end{tabular}

Considering the production of serial end-use products Laminated Object Manufacturing (LOM) as well as 3D-Printing (3DP) are less well-suited. The strength of 3DP for instance lies within the possibility to produce colored samples and prototypes by coloring the binding material. The material of choice is modified gypsum for fullcolored prototypes or silica sand for sand forming tools. The gypsum products have to be infiltrated with an epoxy or wax in order to gain mechanical strength. Building fine detailed products is, due to this low mechanical strength of the binding process, not possible. There are also some systems using metal powder for the application in tooling. These systems, however, are very limited and harder to find.

The so-called Layer Oriented Manufacturing (LOM) uses foil, paper or metal sheets to manufactured product. Each sheet represents one layer. The principle uses laser cutting or stamping tools to contour the sheets, which are then placed on top of each other and adhered. The current significance of this principle is limited. On the other hand, combining this principle with other additive manufacturing principles like Fused Deposition Modeling (FDM) could result in new applications, due to the advantage of LOM in generating relatively big surfaces in a short amount of time. The biggest disadvantage of LOM is the amount of scrap.

All the other principles mentioned in the table currently play a more important role with regards to additive manufactured products. The main reason is the combination of fine detail, handling and the mechanical strength of their products. Most significant for the quality of these products are the following criteria:

- Surface quality (in the direction of the layer as well as alongside the layer set up)

- Mechanical strength within a layer

- Mechanical strength between two layers

- Density of the additive applied material (e.g. air pockets)

This paper will in particular focus on Fused Deposition Modeling (FDM) as the principle of choice for the application.

\section{State-of-the-Art of Fused Deposition Modeling (FDM)}

Fused Deposition Modeling (FDM) uses liquefied or melted thermoplastic material which is applied in layers using minimum one nozzle. The fast cooling of the 
thermo-plastic material itself in the contour applied is responsible for the solidified product. De-pending on the part shape and complexity, a support structure using support material may be required. This is typically the case when the angle of the overhanging contour is bigger than $45^{\circ}$. This support structure is then in a post-processing step removed using either mechanical or thermal force or a bath in which the support material dissolves. The support material is applied by a nozzle just like the product material. This can be either the same nozzle or a second nozzle. All material applied is supplied in a string form run of from a spool. In general, every thermoplastic material can be used for FDM. The current material selection is, however, limited to a few. These include ABS, ABSi, PLA, PC and PPSU. Professional FDM equipment is currently capable of handling a maximum of ten (10) different materials which are only layer-wise or in various mixtures combined. These base materials are also available in a variety of colors increasing the application field. The possibility of layerwise building with different materials and different colors within the same build job or product is responsible for colored single-material or multi-material products.

The size limit of products produced as a single part with no post-gluing of segments is determined by the building volume of the equipment. The largest build volume is currently $914 \mathrm{~mm} \times 610 \mathrm{~mm} \times 914 \mathrm{~mm}$.

The surface quality or roughness is de-fined by the extruded layers. Individual layers vary in thickness between $0,178 \mathrm{~mm}$ and $0,254 \mathrm{~mm}$ and are also visible. If this is an issued from an esthetics standpoint the surface can be smoothed using acetonesteaming afterwards. The new surface is close to that of an injection molded part. Alternatively, FDM products can also be surface coated in order to achieve higher resistance rates or to include other functions of the product.

Tests have shown that the mechanical strength of a FDM product is approx. 80$85 \%$ of a comparable injection molded product.

FDM has one advantage compared to other additive manufacturing technologies. It is easy to stop the process at any giving point in order to place other products or by-products into the product and then continue and finish the building process. Successfully tested examples include metal components shown in figure 1 and even electronics as shown in figure 2. This expands the functionality of additive manufactured products e.g. a lighting function.

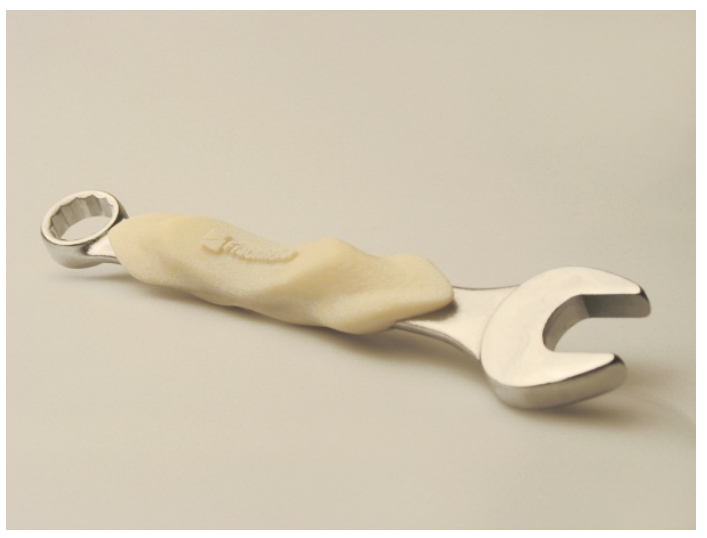

Fig. 1. Embedded wrench I Fraunhofer IPA 


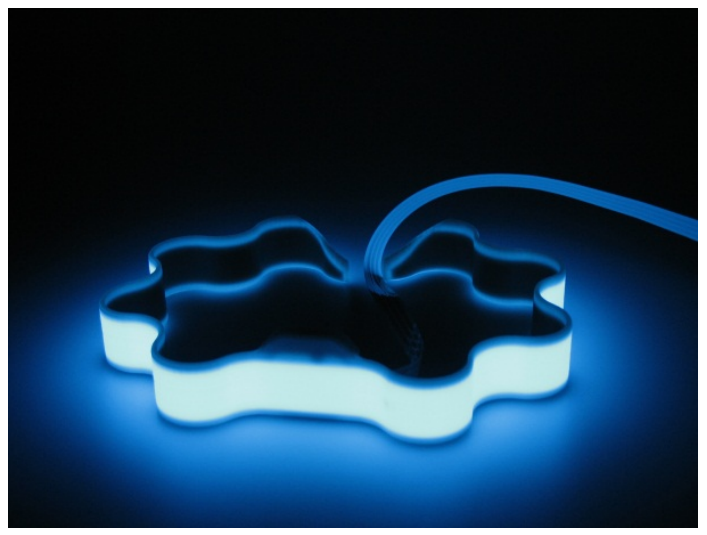

Fig. 2. AM part with integrated EL-Foil and Converter I Fraunhofer IPA

\section{3D Fiber Printer}

\subsection{Basic Mechanical Challenges}

\subsubsection{Fiber Feeding}

Trying to feed a fiber together with a thermoplastic material through an extruder the basic difference between the two be-comes evident immediately. The thermo-plastic filament usually supplied in thick-nesses either $1,75 \mathrm{~mm}$ or $3,0 \mathrm{~mm}$ is able to handle pull and push forces. This circumstance allows the feeding of the filament without bigger issues by using either two gears or using one gear and a ball bearing to restrain the filament. By turning the gear a continuous feed rate is applied, which in turn enables the material to be transported all the way through the heating area and the nozzle to the tip of the nozzle. It is important that during this process of feeding the filament through the nozzle is happening with as little friction as possible. It is also beneficial for the filament to move as straight as possible through the print head unit.

Contrary to the filament, the fibers are unable to handle push forces. In order to move a fiber forward, pull forces have to be applied. Fibers are acting from a mechanical point of view like a true rope, only being able to handle pull forces. Under these circumstances it became clear that the mechanism of feeding the filament forward will not work for the fibers alike.

\subsubsection{Weight and Mass Ratio of Fiber to Thermoplastic Material}

The diameter of the fiber, e.g. a carbon fiber or a carbon fiber bundle, is much less than the diameter of the thermoplastic filament. The thickness of a $1 \mathrm{k}$ carbon fiber cable for instance is only $190 \mu \mathrm{m}$. This is also the reason that the carbon fiber cannot be transported by the molten thermoplastic material, simply by pulling it along. Assuming a round cross section of the fiber its cross sectional area calculates to approximately $\mathrm{A}_{\text {Fiber }}=28.300 \mu \mathrm{m}^{2}$. The cross sectional area of a $0,5 \mathrm{~mm}$ diameter nozzle is in comparison $\mathrm{A}_{\text {nozzle }}=196.000 \mu \mathrm{m}^{2}$. The cross sectional area of a $1,75 \mathrm{~mm}$ thick filament finally is $\mathrm{A}_{\text {Filament }}=2.405 .000 \mu \mathrm{m}^{2}$. 
In order to completely fill the nozzle with fiber and thermoplastic material, the later has to fill the remaining cross sectional area of $A_{\text {nozzle }}-A_{\text {Fiber }}=167.700 \mu \mathrm{m}^{2}$.

Assuming the same feed rate for the fiber as well as the filament the nozzle will be receiving $2.405 .000 \mu \mathrm{m}^{2}$, which is more than 14 times the amount of the remaining cross sectional area. The effect would be excess material resulting in a clogged up nozzle.

This basic and simple calculation brought up the idea of embedding the fibers prior to it entering the extruder. This idea would have been a much easier task to complete. The fibers could have been equally applied with the thermoplastic material in an external apparatus.

In order to solve the problem stated above, a filament string with the exact diameter of the nozzle would need to be manufactured. Considering the mentioned example a $0,5 \mathrm{~mm}$ thick filament string of thermo-plastic material including the fiber already would be needed. Using such a theoretically and practically possible filament would result in the same problems as the fibers itself. The filament would be too weak to handle the push forces required for the FDM process and the nozzle to work.

\subsection{Solution}

The problems discussed under point 3.1.1 and 3.1.2 can be reduced and simplified to the problem of the fiber feeding process. The fiber(s) and the filament have to be run at different feed rates and therefore it is impossible to feed the fibers with the filament using the same transportation unit. As mentioned above, transporting the filament is not an issue and can be considered state-of-the-art. To feed the fiber(s) at the matching ration of the feed rate of the filament is the challenge. To reduce the complexity of finding and running the matching feed rate of the fiber, one idea was to fix the endless fiber directly on the building platform prior to starting the building process. This procedure would then automatically assure that the fiber is applied depending on the feed rate of the extruder head. Proceeding this way, however, will cause other problems. One being the fact that the fiber would need to be manually attached, the other being that the fiber and with it the matrix already produced will be under constant force and therefore additional stress would be put on the part during the build process. This issue comes especially into effect when the head has to move in a curvature or moving into a corner (depending on the geometry of the product) instead of a straight line. Describing a curvature or running into a corner applies strong shear forces to the fiber-matrix so that the risks of delamination or pull-off are increasing. Additionally the strong bending of the fiber at the nozzle tip poses the risk of dam-aging the fibers as well as an increased wear of the nozzle itself. These two risks are especially high due to the dynamic pull forces with which the fibers are pulled through the nozzle.

With these issues and risks in mind the search for a solution ended in the modified application of the water-jet pump principle.

This principle is characterized through the fact that a flowing medium is guided through a pipe and within that pipe along an opening. The flow of the medium is creating suction and thereby pulling the medium from the opening. This principle cannot be copied one to one to fiber and filament but requires adjustments. On the one hand, a very slow moving medium like the molten thermo-plastic material does not 
create suction. On the other hand, such a thin fiber would not be moved by suction. These issues are solved by the design of the 3D Fiber Printer nozzle. The fiber must be fed through a side channel into the main channel in which the filament is fed. The position of the meeting point of the two channels has to be exactly at the point where the diameter of the nozzle has reached the $0,5 \mathrm{~mm}$. The extruder nozzle is designed in the way that the molten filament is fed through several $\mathrm{mm}$ of length through the channel with the diameter of $0,5 \mathrm{~mm}$. Within these few millimeters the fiber is introduced to the molten filament and "adheres" to it forming the mechanical bond. This bond will now pull the fiber along with the molten material achieving the right mix ratio of thermoplastic material to fiber to create the best embedding of the fiber as well as the correct feed rate of both.

\subsection{Design of the 3D Fibre Print Nozzle}

The basis for the design of this special nozzle is the standard design of conventional brass nozzle in order to realize the same simple exchange mechanism of the nozzles within the print head. An additional side channel is designed meeting the main channel at a specific point. The fibers are introduced through this channel. The design and size of this channel are too small to be manufactured with conventional technologies. The solution is manufacturing this nozzle by the additive manufacturing technology of Selective Laser Melting (SLM). The side channel is also forming a curve see figure 3. This curve is needed to smooth the entrance of the fiber into the main channel and by that reducing the wear of the nozzle at the meeting point during the use of the nozzle. Car-bon fibers are known for acting like sanding paper to any burr or hard break resulting in the destruction of the nozzle in the long run.

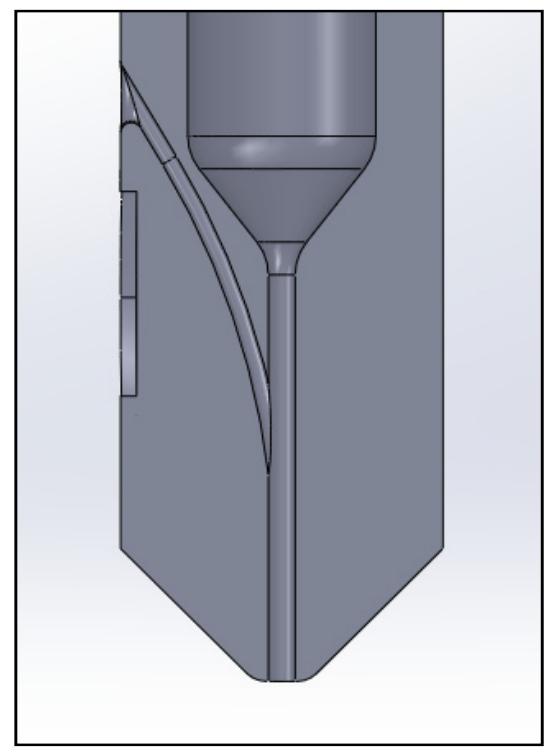

Fig. 3. FDM 3D Fiber Printer nozzle design proposal including a curved fiber channel Fraunhofer IPA 


\section{$4 \quad$ First Test Results}

The first test runs were performed using the designed and built nozzle together with a $3 \mathrm{~K}$ carbon fiber and a thermoplastic elastomer from the urethane group from Bayer Material Science. Starting point was the application of a straight line, to test the complete embedding of the fiber with the molten filament see figure 4 . The base plate for the test was a heated aluminum plate with the temperature set to $110^{\circ} \mathrm{C}$. For adhesion purposes a kapton adhesive tape was applied to the aluminum plate. The kapton adhesive tape is a high heat resistance adhesive tape which also shows good adhesion to the urethane material.

The first test results showed success at the application and feeding of both materials in the right setup. The visual inspection showed a good embedding of the fiber within the product.

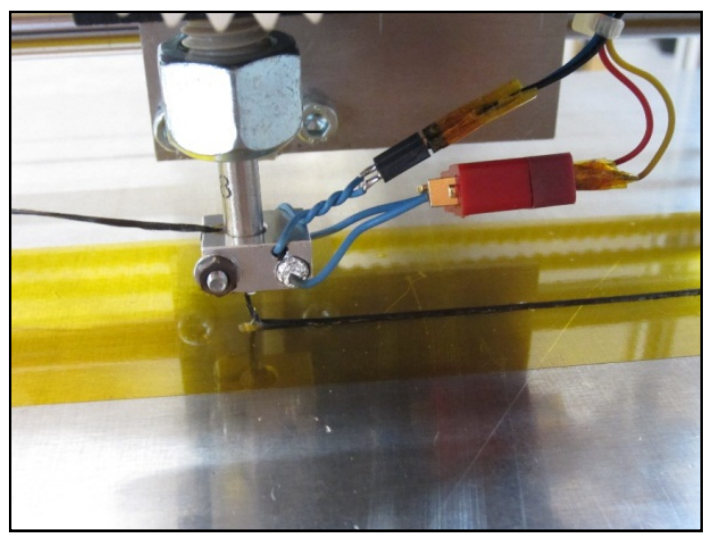

Fig. 4. First printing test with new nozzle and fiber included I Fraunhofer IPA

\section{$5 \quad$ Next Steps and Outlook - 3D Fiber Printer}

The industrial implementation of the de-veloped technology requires further optimization and tests. These tests include e.g. pull tests to test the matrix.

It was, however, shown that the fibers can be embedded into products manufactured with the Fused Deposition Modeling (FDM) technology.

Besides the testing of the adhesion forces between the thermoplastic material and the fibers, which have to include chemical testing, the long-term testing of the nozzle and its design for wear as well as the feeding mechanism are the next steps planned. The later tests are also intended to determine whether SLM is the right technology to manufacture the nozzles and whether the SLM manufactured nozzles are suited for industrial use. 


\section{References}

1. Jäger, et al.: Carbonfasern und ihre Verbund-werkstoffe: Herstellungsprozesse, Anwendungen und Marktentwicklung. Die Bibliothek der Technik, Band 326. Verlag Moderne Industrie (2010)

2. Flemming, et al.: Faserverbundbauweisen, Fasern und Matrices. Springer-Verlag (1995)

3. Flemming, et al.: Faserverbundbauweisen, Halbzeuge und Bauweisen. Springer (1996)

4. Flemming, et al.: Faserverbundbauweisen, Fertigungsverfahren mit duroplastischer Matrix. Springer (1999)

5. Grießbach: Praxis Rapid Technologien, Handbuch für Produktentwickler, Techniker, Kaufleute. Vonroth \& Bode KG Verlag (2009)

6. VDI-Richtlinie: Generative Fertigungsverfahren - Rapid-Technologien (Rapid Prototyping) - Grundlagen, Begriffe, Qualitätskenngrößen, Liefervereinbarungen. VDI, Düsseldorf (2009)

7. Zäh, M.: Wirtschaftliche Fertigung mit Rapid-Technologien: Anwender-Leitfaden zur Auswahl geeigneter Verfahren. Carl Hanser Verlag (2006) 